\title{
Uber die Abbildungseigenschaften magnetischer Sektorfelder bei Berücksichtigung des Streufeldes
}

\author{
Von L. A. König * und H. Hintenberger \\ Aus dem Max-Planck-Institut für Chemie, Mainz \\ (Z. Naturforschg. 10 a, 877-886 [1955] ; eingegangen am 10. September 1955) \\ Josef Mat ta $\mathrm{s}$ ch zum 60. Geburtstag gewidmet
}

\begin{abstract}
Nach einer experimentellen Untersuchung über den Streufeldverlauf am Rande der Polschuhe verschiedener Magnete, bei der auch der Einfluß von Eisenblenden zur Abschirmung der Streufelder eingehend untersucht wird, werden die Abbildungseigenschaften magnetischer Sektorfelder mit Berücksichtigung des Streufeldes für Ionenstrahlen in der Mittelebene des Feldes rechnerisch behandelt. Im Anschluß an eine frühere Arbeit ${ }^{1}$, bei der die Linsengleichung und die Lateralvergrößerung unter Berücksichtigung der Streufelder untersucht wurde, werden Ausdrücke für die Dispersion und den Öffnungsfehler magnetischer Sektorfelder für geradlinige und gekrümmte Feldbegrenzungen angegeben. Um den Einfluß der Streufelder zahlenmäßig zu demonstrieren, werden mit den erhaltenen Formeln einige Beispiele durchgerechnet, wobei, entsprechend den Resultaten der Streufeldmessungen, die Grenze zwischen dem homogenen Feldteil und dem Streufeld um die Strecke $\left|Z_{\mathrm{s}}\right|=1,5 k$ innerhalb des Polschuhrandes und parallel zu ihm angenommen wird, während für die durchgemessenen Magnete die Begrenzung des homogenen Ersatzfeldes, das zur gleichen Abbildung wie das wirkliche Feld führt, etwa um die Strecke $Z_{\mathrm{E}}=2,1 k$ außerhalb des Polschuhrandes verläuft $(2 k=$ Polschuhabstand). Die Unterschiede, die sich bei der Berechnung der Dispersion und des Öffnungsfehlers aus den allgemeinen Formeln mit Berücksichtigung des Streufeldes und aus den Formeln für das homogene Ersatzfeld für Strahlen in der Symmetrieebene ergeben, hängen stark von der Dimensionierung des Magneten ab. So beträgt z. B. die Abweichung der exakt berechneten Größen von den mit Hilfe eines homogenen Ersatzfeldes berechneten bei Magneten, wie sie bei doppelfokussierenden Apparaten verwendet werden, nur wenige Prozent, während sie bei den in Massenspektrometern oder Massentrennern üblichen Magneten viel größer werden können.
\end{abstract}

$\mathrm{B}$ ei der Behandlung der ionenoptischen Eigenschaften magnetischer Sektorfelder wird in der Regel mit rein homogenen Magnetfeldern gerechnet $^{2}$. Ein von den Polschuhrändern begrenztes homogenes magnetisches Sektorfeld hat jedoch nicht dieselben Ablenk- und Abbildungseigenschaften wie das wirkliche Magnetfeld, so daß eine Berücksichtigung des Streufeldes auf jeden Fall notwendig ist. Man kann bei der Berechnung der Abbildungseigenschaften das wirkliche Magnetfeld durch ein ideales homogenes Feld geeigneter Dimensionierung ersetzen. Dabei ist es aber nur möglich, durch geeignete Dimensionierung eine Eigenschaft des Ersatzfeldes (z. B. den Ablenkwinkel) dem des wirklichen Feldes genau anzupassen. Es soll das Ziel dieser Arbeit sein, für die Dispersion und den Öffnungsfehler für Strahlen, die in der Mittelebene verlaufen, allgemein gültige geschlossene Ausdrücke abzuleiten, bei denen die Wirkung der Streufelder voll in Rechnung gesetzt worden ist, und sie mit den Ausdrücken zu vergleichen, die sich für dieselben Größen unter Zuhilfenahme eines homogenen Ersatzfeldes geeigne-

* Dissertation Mainz 1955 (D 77).

1 L. A. Kö n i g, Z. Naturforschg. 10a, 652 [1955].

2 Siehe z. B. H. Ewald u. H. Hint e n b e rger, Methoden und Anwendungen der Massenspektroskopie, Verlag Chemie, Weinheim/Bergstraße, 1953. ter Dimensionierung ergeben. Damit die durch das Streufeld wirklich auftretenden Einflüsse auch quantitativ abgeschätzt werden können, sind charakteristische Streufelder an oft benutzten Magnettypen experimentell sorgfältig ausgemessen worden.

In den Rechnungen wird der Feldverlauf offen gelassen, d. h. es wird die zur Symmetrieebene senkrechte Komponente der Kraftflußdichte $B_{x}$

$$
B_{x}=B_{0} h(\mathfrak{r})
$$

angesetzt, worin $B_{0}$ der Wert der Kraftflußdichte im homogenen Teil des Feldes und $h(\mathfrak{r})$ eine Funktion des Ortsvektors $\mathrm{r}(0 \leqq h(\mathfrak{r}) \leqq 1)$ sein soll.

Arbeiten, in denen der Einfluß der Streufelder auf die ionenoptischen Abbildungseigenschaften magnetischer Sektorfelder z. Tl. auch außerhalb der Symmetrieebene, in dieser jedoch weniger allgemein, weniger genau oder den massenspektroskopischen Anwendungen weniger angepaßt behandelt wurden, sind bereits von anderen Autoren, z. B. von Her zog ${ }^{3}, \mathrm{Cotte}^{4}$, Marschall ${ }^{5}$, Coggeshall ${ }^{6}$,

${ }^{3}$ R. H e rz o g, Z. Phys. 97, 596 [1935].

4 M. C o t t e, Thèses Univ. Paris 1938, Ser. A, No. 1807, No. D'ordre 2673.

5 H. M a r s c h a l l, Phys. Z. 45, 1 [1944].

6 N. D. Cog g e s ha l l, J. Appl. Phys. 18, 855 [1947]. 
Ploch und Walcher ${ }^{7}$, Sturrock ${ }^{8}$ und von R e u terswär d ${ }^{9}$ durchgeführt worden.

\section{Streufeldmessungen}

Die Messungen dienten der Bestimmung von $h(\mathfrak{r})$, insbesondere bei Anwendung einer Eisenblende. Sie wurden nach folgenden Verfahren durchgeführt:

1. Messung mit der Induktionsspule,

2. Messung im elektrolytischen Trog.

Die Induktionsspulen wurden teils mit einer Vorrichtung von Hand um $180^{\circ}$ gekippt, teils durch einen Synchronmotor gedreht und die mit einem Kommutator gleichgerichteten Ströme mit einem Spiegelgalvanometer gemessen. Die Spulengröße lag zwischen $5 \mathrm{~mm} \phi, 4 \mathrm{~mm}$ Länge und 1,5 $\mathrm{mm} \phi$, $1 \mathrm{~mm}$ Länge. Die Nachweisempfindlichkeit war so hoch, daß bei $50 \mathrm{~cm}$ Lichtzeigerlänge durch das Erdfeld mehrere $\mathrm{mm}$ Ausschlag erzielt wurden.

Der elektrolytische Trog wurde in der üblichen Weise (vgl. z. B. ${ }^{10}$ ) aufgebaut. Die Äquipotentiallinien wurden mit Hilfe eines Pantographen von der Oberfläche des Elektrolyten auf den Zeichentisch übertragen.

Aus je zwei Äquipotentiallinien wurde $h(z)$ nach der in zweiter Näherung gültigen Formel

$$
h(z)=\frac{1}{x_{2}^{2}-x_{1}^{2}}\left[x_{2}^{2} \frac{x_{01}}{x_{1}}-x_{1}^{2} \frac{x_{02}}{x_{2}}\right]
$$

berechnet, welche aus potentialtheoretischen Überlegungen folgt. Hierin bedeutet $x_{0 i}$ den Abstand der Äquipotentiallinie $i$ im homogenen Teil des Feldes

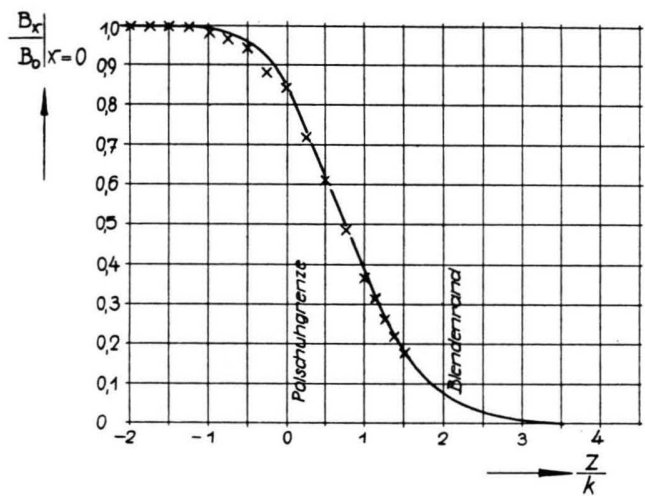

Abb. 1. Streufeldverlauf bei ebenen Polschuhen mit dicker Eisenblende (vgl. Abb. 5). Symmetrische Anordnung: $d=2 b$ $=2 \mathrm{k}$. Die ausgezogene Kurve wurde direkt mit Induktionsspule gemessen; die eingezeichneten Punkte sind nach der im Text genannten Formel aus den im elektrolytischen Trog am Äquipotentiallinien nach der im Text genannten Formel berechnet.

7 W. Ploch u. W. W a lch er, Z. Phys. 127, 274 [1950]. 8 P. A. Sturrock, Proc. Phys. Soc., Lond. 63, 954 [1950]. 9 C. Reuterswärd, Ark. Fys. 3, 53 [1951]; 4, 159 [1951].

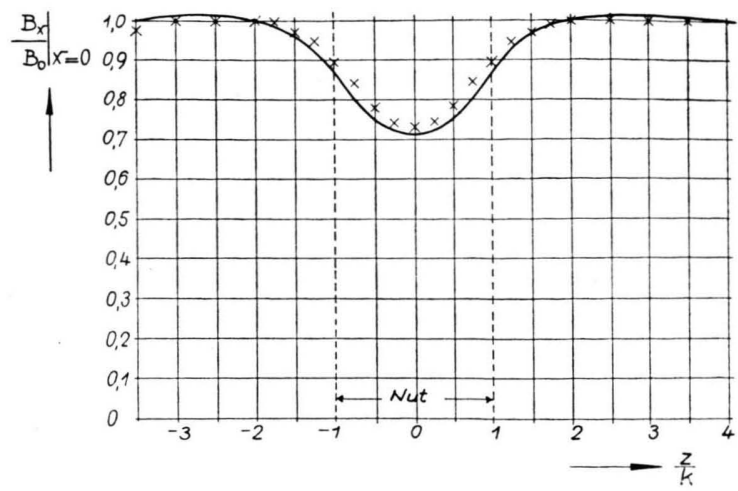

Abb. 2. Streufeldverlauf in der Umgebung der Plattenhalternut (s. Abb. 3) eines Massenspektrograph-Magneten (M a t $\mathrm{t}$ a u c h-H e r z o g scher Typ). Die ausgezogene Kurve ist direkt mit Induktionsspule gemessen; die eingezeichneten Punkte sind aus den im elektrolytischen Trog aufgesuchten Äquipotentiallinien nach der im Text genannten Formel berechnet.

von der Symmetrieebene des Elektrodenmodells, $x_{i}$ den Abstand der Äquipotentiallinie von der Symmetrieebene am Ort $z$ ( $z=$ Koordinate senkrecht zur Polschuhstirnfläche; siehe z. B. Abb. 10).

Die Ergebnisse der Messungen im elektrolytischen Trog stimmten mit den auf direktem, magnetischem

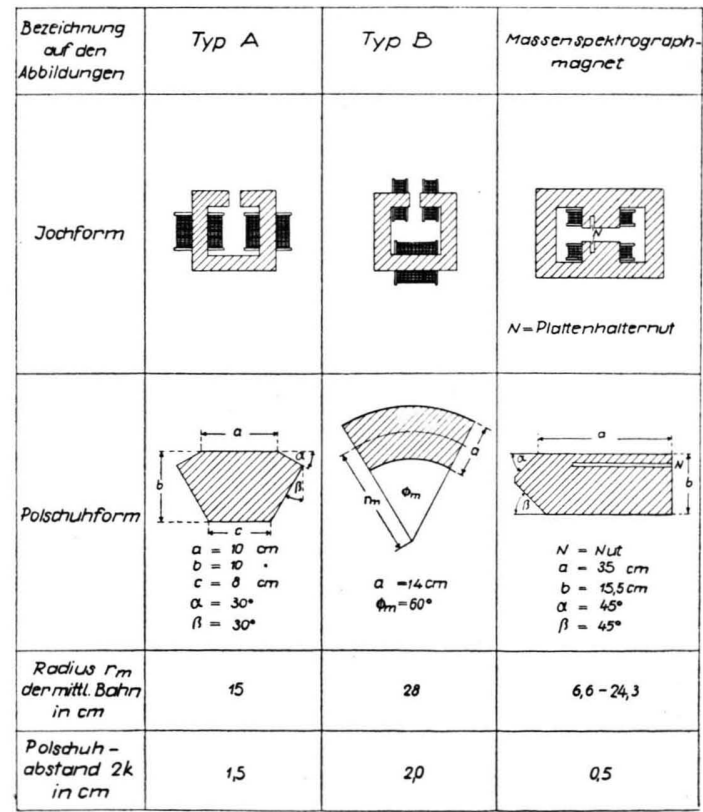

Abb. 3. Ubersicht über die Magneten, bei denen der Feldverlauf bestimmt wurde.

${ }^{10} \mathrm{Z}$ w orykin, Morton, Ra mberg, Hillieru. $\mathrm{V}$ ance, Electron Optics and the Electron Microscope, New York 1945. 


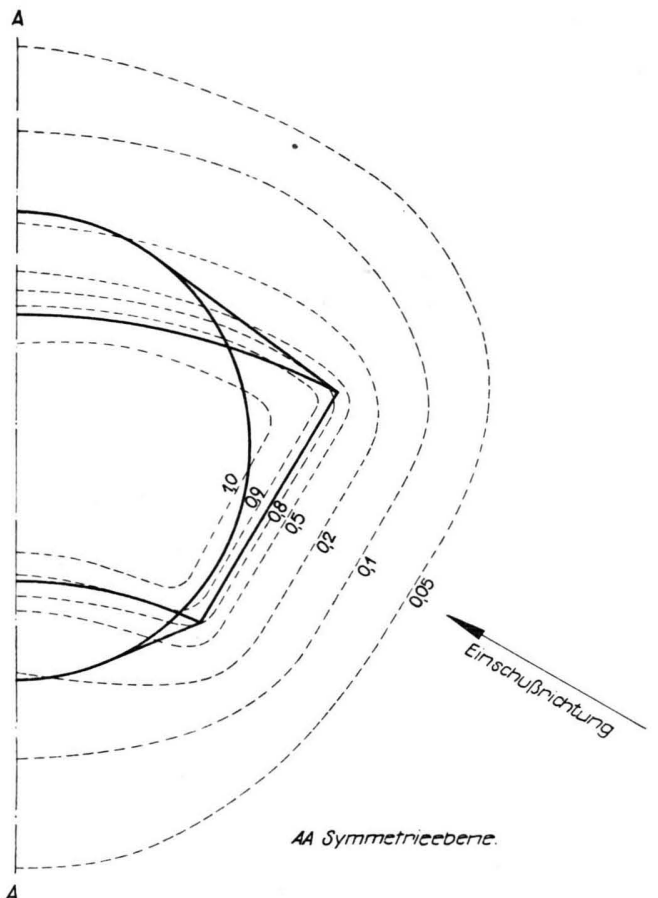

Abb. 4. Linien gleicher Feldstärke im Felde des Magneten B.

Weg gewonnenen Resultaten gut überein. Die Abb. 1 und 2 enthalten hierfür zwei Beispiele.

Die Messungen wurden an drei verschiedenen Magnettypen durchgeführt, über die nähere Einzelheiten in Abb. 3 enthalten sind. Abb. 4 gibt die Linien gleicher Feldstärke am Magneten B wieder. Abb. 2 zeigt den Feldverlauf in der Plattenhalternut des Magneten eines Massenspektrographen vom M a t a u c h - Her z o g schen Typ.

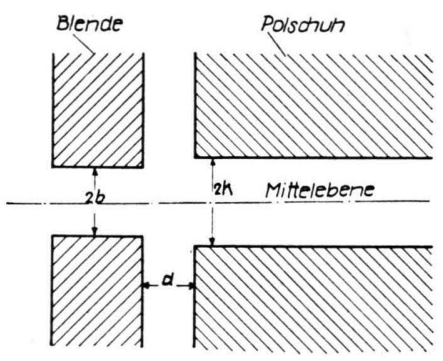

Abb. 5. Eisenblende. $2 k$ Polschuhabstand, $2 b$ Blendenweite, $d$ Blendenabstand.

Die Meßergebnisse über die Wirkung einer Eisenblende (Abb.5), die zur Abschirmung des Streufeldes angebracht wird, sind in Abb. 6 und 7 zusammengefaßt. Abb. 6 zeigt die größte Steilheit des Feldabfalles $\operatorname{tg} \alpha_{\max }$ und Abb. 7 den Abstand $Z_{\mathrm{h}}$ des
Punktes, wo die Kraftflußdichte auf $1 / 2 B_{0}$ abgesunken ist, von der Polschuhgrenze in Abhängigkeit vom Blendenabstand $d$ für verschiedene Werte der halben Blendenweite $b$. (Als Längeneinheit wird dabei stets der halbe Polschuhabstand $k$ genommen,

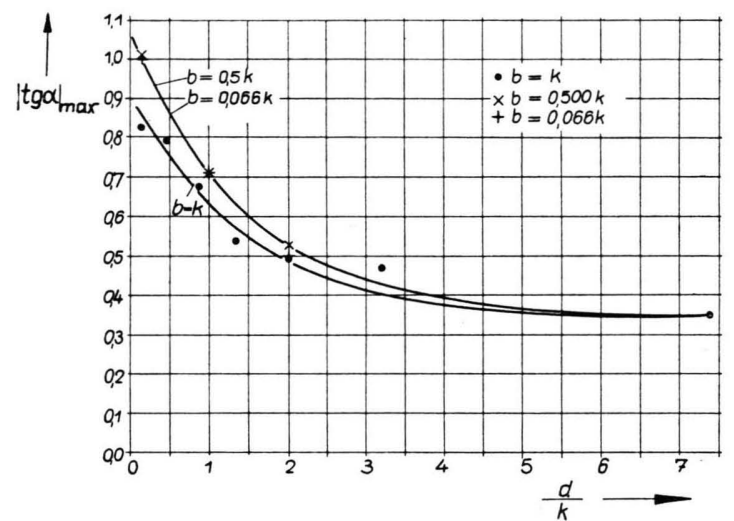

Abb. 6. Absoluter Betrag tg $\alpha_{\max }$ der größten Steilheit des Feldabfalles als Funktion des Blendenabstandes $d$. Halbe Blendenweite $b$ als Parameter. Der halbe Polschuhabstand $k$ wurde als Längeneinheit benutzt. Die Meßpunkte stammen teils aus direkten Magnetfeldmessungen, teils aus Messungen im elektrolytischen Trog.

s. Abb. 5.) Als besonders bemerkenswert ist hervorzuheben, daß die Grenze zwischen Streufeld und dem homogenen Teil des Feldes durch die Blende kaum beeinflußt wird.

Am Ort $Z_{\mathrm{s}}=-1,5 k$ wird fast unabhängig von der Lage und Ausdehnung der Blende eine Kraft-

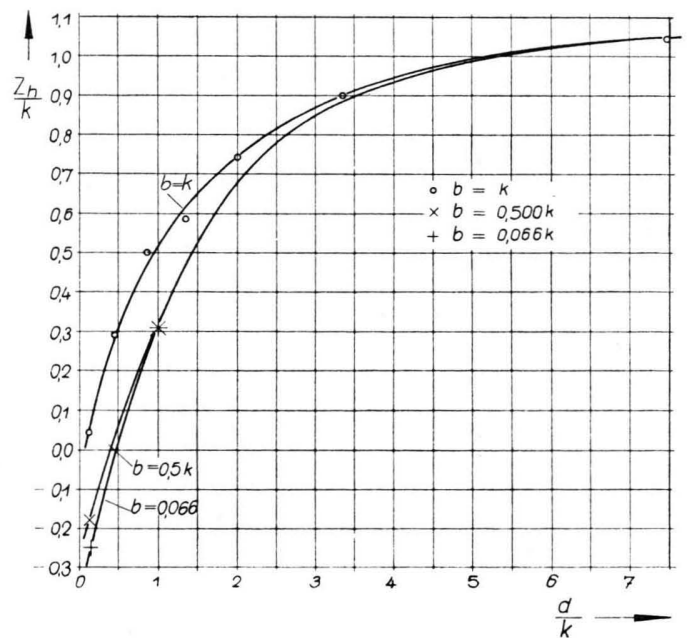

Abb. 7. Ort $Z_{\mathrm{h}}$, an dem die Feldstärke auf die Hälfte des Wertes im homogenen Feld abgesunken ist. $Z_{\mathrm{h}}$ ist vom Polschuhrand aus gemessen und nach außen positiv gezählt. Der halbe Polschuhabstand wurde als Längeneinheit benutzt. Die Meßpunkte stammen teils aus direkten magnetischen Messungen, teils aus Messungen im elekktrolytischen Trog. 
flußdichte erreicht, die sich von der im homogenen Feld herrschenden um weniger als $1 \%$ unterscheidet. Dort kann für die Rechnung dann auch die Grenze zwischen homogenem Feld und Streufeld angenommen werden. Durch die Eisenblende wird aber die Steilheit des Feldabfalles, die Halbwertskoordinate $Z_{\mathrm{h}}$ und natürlich der Feldverlauf innerhalb der Blende stark beeinflußt. (S. Abb. 1, 6 u. 7.)

Für das Folgende sei hervorgehoben, daß bei Magneten mit im Vergleich zum Polschuhabstand weit ausgedehnten geradlinigen Polschuhgrenzen, wie sie bei Massenspektrometern und Massenspektrographen vielfach gebräuchlich sind, die Linien gleicher Feldstärke in der Nähe der Polschuhstirnfläche parallele Geraden darstellen.

Abb. 8 gibt die Meßergebnisse verschiedener Autoren für den Abfall des Feldes am Rande der Polschuhe im Vergleich zu einer von Thom son ${ }^{11}$

11 J. J. Thomson, Recent Researches in Electricity and Magnetism, Oxford 1893.

${ }_{12}$ Mit Hilfe der Meßergebnisse kann man leicht einige für Magnetberechnungen wichtige Formeln zahlenmäßig auswerten: Setzt man den Gesamtkraftfluß $\Psi$ im Luftspalt des Magneten $\Psi=\Psi_{\mathrm{n}}+\Psi_{\mathrm{s}}\left(\Psi_{\mathrm{n}}=F B_{0} \quad\right.$ Nutzfluß, $F$ Polschuhfläche, $\Psi_{\mathrm{s}}=$ Streufluß), so findet $\operatorname{man} \Psi_{\mathrm{s}}=1,22 \mathrm{DU} B_{0}$ durchgeführten Rechnung wieder. Die Messungen der Verfasser wurden getrennt an drei verschiedenen Massenspektrometer-Magneten (zwei vom Typ A, einer vom Typ B) durchgeführt.

Der Feldverlauf erwies sich innerhalb des Bereiches von 500 bis $7000 \mathrm{Gauß}$ von $B_{0}$ unabhängig. Bei kleineren Kraftflußdichten wird das Feld durch einen bearbeitungsabhängigen Remanzeffekt merklich inhomogen. (Vgl. Abb. 9, wo $B_{0}$ so gewählt wurde, daß der Feldabfall außerhalb der Polschuhe ungeändert blieb) ${ }^{12}$.

\section{Die Theorie der ionenoptischen Abbildung in der Symmetrieebene des magnetischen Sektor- feldes bei Berücksichtigung des Streufeldes}

Die Berechnung der Abbildungseigenschaften läßt sich für die Symmetrieebene völlig allgemein durch-

( $D$ Polschuhabstand, $U$ Polschuhumfang). In l.c. ${ }^{2}$ war auf S. 126 auf Grund einer weniger genauen Abschätzung statt 1,22 ein kleinerer Faktor angegeben worden.

Ebenso kann man die maximale im Luftspalt erreichbare Kraftflußdichte $B_{0}$ abschätzen:

$$
B_{0}=Q B_{Q} /(F+1,22 D U)
$$

( $Q$ kleinster Jochquerschnitt, $B_{Q}=$ Kraftflußdichte im kleinsten Jochquerschnitt $Q$ ).

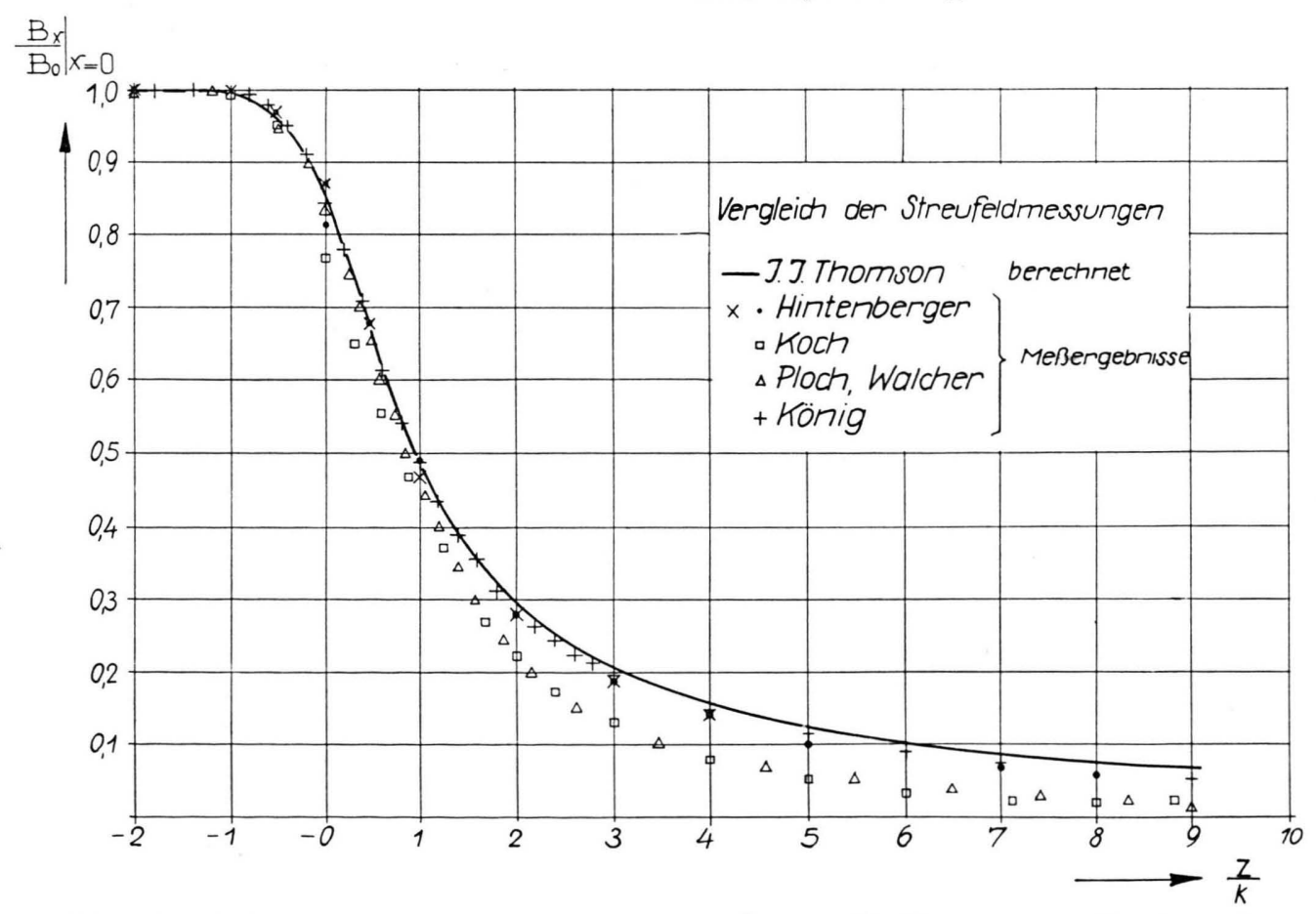

Abb. 8. Vergleich der Streufeldmessungen verschiedener Autoren. Die Messungen von K o c h wurden an einem Magneten mit kreisförmigem Polschuhquerschnitt durchgeführt. Die übrigen Kurven, auch die von Th o m s o n berechnete, beziehen sich alle auf Polschuhe mit ebener Stirnfläche.

Polschuhrand bei $Z / k=0$. 


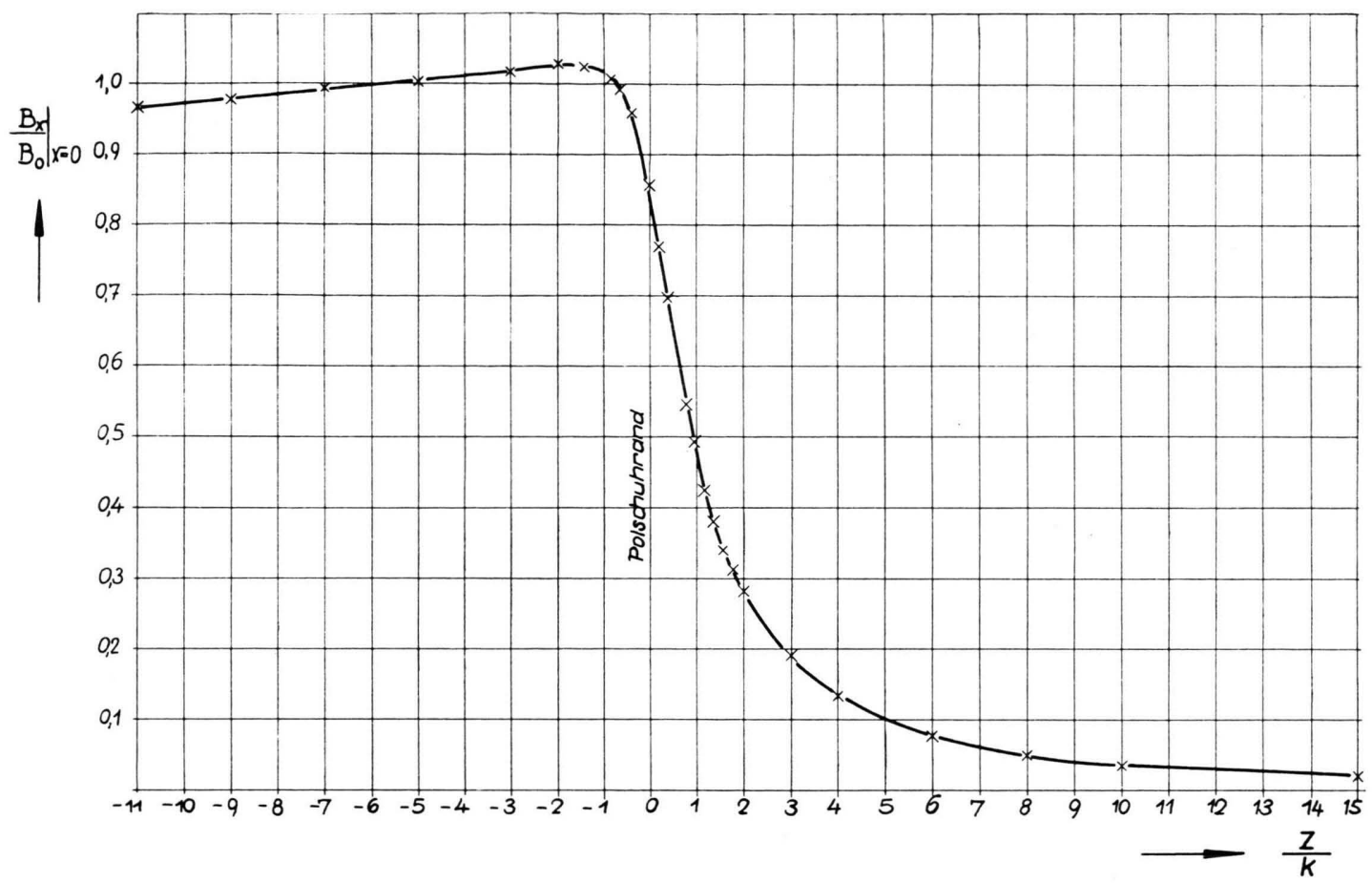

Abb. 9. Remanzeffekt am Magneten B, Messung in Einschußrichtung (vgl. Abb. 3). $B_{0} \approx 170$ Gauß.

führen, d. h. man kann die Ergebnisse für beliebigen Streufeldverlauf formulieren ${ }^{1}$. Da die Rechnungen viel Platz beanspruchen, wollen wir in dieser Arbeit Ableitungen nur kurz andeuten und uns im Wesentlichen auf die Wiedergabe der Ergebnisse für zwei oft benutzte Spezialfälle beschränken:
Fall 1: Gerade, im Vergleich zum Polschuhabstand weit ausgedehnte Polschuhgrenzen, bei denen $B_{x}$ nur von der kartesischen Koordinate $z_{1}$ bzw. $z_{2}$ senkrecht zur Polschuhbegrenzung abhängt (vgl. Abb. 10).

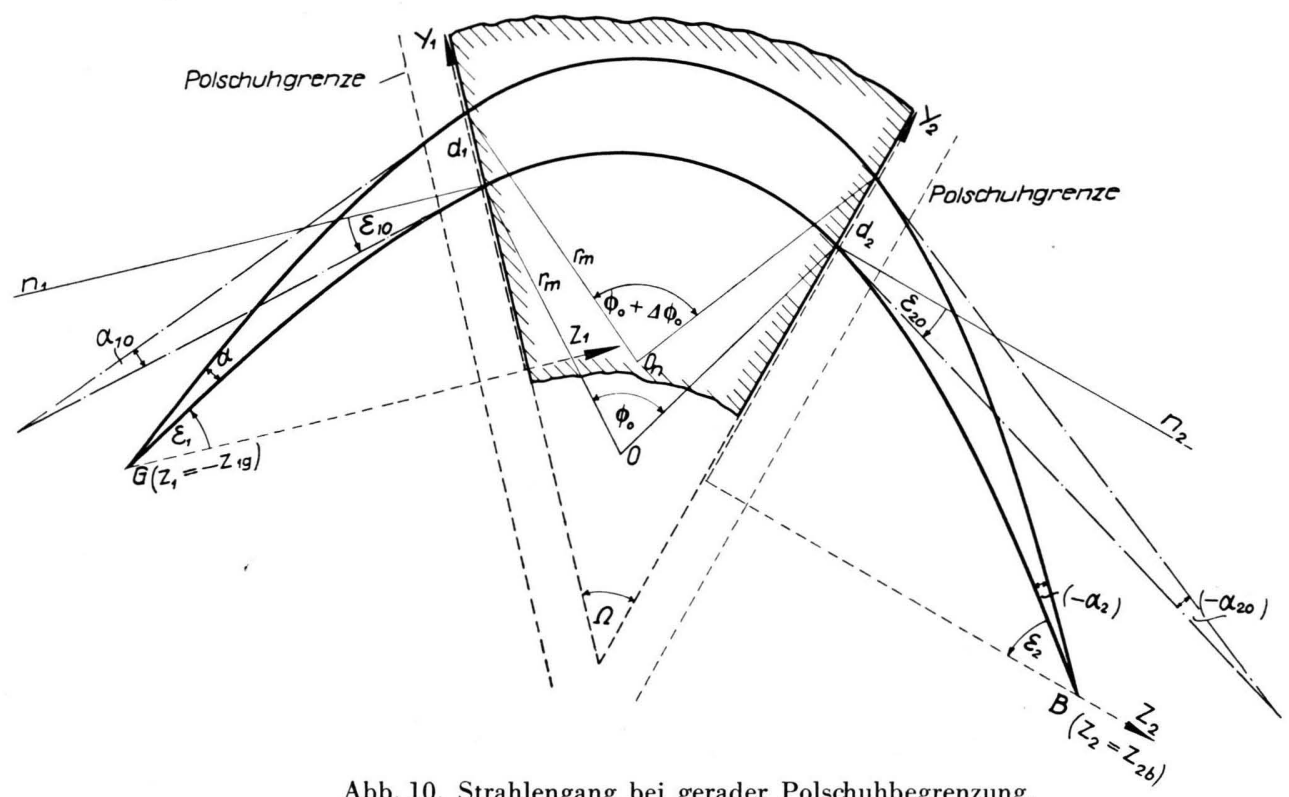

Abb. 10. Strahlengang bei gerader Polschuhbegrenzung. 
Fall 2: Konvex ${ }^{13}$ gekrümmte Polschuhbegrenzungen, bei denen die Feldstärke nur von den Abständen $r_{1}$ oder $r_{2}$ von den gegenstandseitigen bzw. bildseitigen Krümmungsmittelpunkten $\mathrm{M}_{1}$ oder $\mathrm{M}_{2}$ der Polschuhbegrenzung, die dem Ein- bzw. Austrittspunkt des Mittelstrahles zugeordnet sind, abhängt (vgl. Abb. 11).

Diese Beschränkungen sind durch die Ergebnisse der Streufeldmessungen in nicht zu großer Entfernung vom Polschuhrand gerechtfertigt. Da das Streufeld in Polschuhnähe am wirksamsten ist, sind dabei quantitativ gut brauchbare Ergebnisse zu erwarten. Geht die Bahn durch Feldbereiche, wo keine der obengenannten Voraussetzungen erfüllt ist, kann man entweder auf die allgemeineren Formeln zurückgreifen, die noch in einer ausführlicheren Arbeit angegeben werden sollen, oder für $h(\mathfrak{r})$ einen Feldverlauf ansetzen, der dem tatsächlichen Bahnverlauf angepaßt ist.

Wie bereits gezeigt wurde ${ }^{1}$, kann die für den rein homogenen Teil des Sektorfeldes gültige Abbildungsgleichung

$$
\left(l_{1}^{*}-g_{10}\right)\left(l_{2}{ }^{*}-g_{20}\right)=f_{0}{ }^{2}
$$

zur Ermittlung des Zusammenhanges zwischen den Abständen $z_{1 \mathrm{~g}}$ des Gegenstandes und $z_{2 \mathrm{~b}}$ des Bildes von der entsprechenden Grenze des homogenen Feldteiles (s. Abb. 10 u. 11) auch dann herangezogen werden, wenn Streufelder vorhanden sind, die sich bis an den Gegenstands- und den Bildort erstrecken. Die Größen $g_{10}, g_{20}$ und $f_{0}$ beziehen sich auf den rein homogenen Feldteil und sind gegeben durch

$$
\begin{aligned}
& \text { a) } g_{10}=r_{\mathrm{m}} \frac{\cos \varepsilon_{10} \cos \left(\Phi_{0}-\varepsilon_{20}\right)}{\sin \Omega}, \\
& \text { b) } g_{20}=r_{\mathrm{m}} \frac{\cos \varepsilon_{20} \cos \left(\Phi_{0}-\varepsilon_{10}\right)}{\sin \Omega}, \\
& \text { c) } f_{0}=r_{\mathrm{m}} \frac{\cos \varepsilon_{10} \cos \varepsilon_{20}}{\sin \Omega}, \quad\left(\Omega=\Phi_{0}-\varepsilon_{10}-\varepsilon_{20}\right) \text {. }
\end{aligned}
$$

Zwischen den Größen $l_{i}^{*}$ und $z_{1 \mathrm{~g}}$ bzw. $z_{2 \mathrm{~b}}$ bestehen die Beziehungen

$$
l_{i}^{*}=\left.\cos \varepsilon_{i 0} \frac{\partial y_{i \mathrm{~B}}}{\partial \varepsilon_{i 0}}\right|_{z_{i}=0}\left(y_{i \mathrm{~B}} \text { Bahnordinate }\right),
$$

die für gerade Feldgrenzen durch

$$
\begin{array}{r}
l_{i}^{*}=\cos ^{2} \varepsilon_{i 0} \int_{a_{i}}^{b_{i}} \frac{\mathrm{d} \zeta}{\left(1-f_{i}^{2}(\zeta)\right)^{3 / 2}} \\
\left(a_{1}=-z_{1 \mathrm{~g}}, b_{1}=0 ; a_{2}=0, b_{2}=z_{2 \mathrm{~b}}\right)
\end{array}
$$

${ }^{13}$ Bei konkaven Feldgrenzen ist die Abhängigkeit des Feldes von nur einer Koordinate nicht so leicht realisierbar. gegeben sind mit

$$
f_{i}=-\frac{1}{r_{\mathrm{m}}} \int_{0}^{\zeta} h_{i}\left(\zeta^{\prime}\right) \mathrm{d} \zeta^{\prime}+(-1)^{i+1} \sin \varepsilon_{i 0},
$$

und die für gekrümmte Feldgrenzen durch

$$
\begin{gathered}
l_{i}^{*}=R_{i} \cos \varepsilon_{i 0} \frac{\int_{R_{i}}^{r_{i 0}} \frac{r \mathrm{~d} r}{\left(r^{2}-f_{i}{ }^{2}(r)\right)^{3 / 2}}}{\frac{1}{R_{i} \cos \varepsilon_{i 0}}-\int_{R_{i}}^{r_{i 0}} \frac{r \mathrm{~d} r}{\left(r^{2}-f_{i}{ }^{2}(r)\right)^{3 / 2}}} \\
\left(r_{10}=r_{1 g}, r_{20}=r_{2 \mathrm{~b}}\right)
\end{gathered}
$$

mit $f_{i}=\frac{1}{r_{\mathrm{m}}} \int_{R_{i}}^{r} h_{i}(\varrho) \varrho \mathrm{d} \varrho+R_{i} \sin \varepsilon_{i 0}$

dargestellt werden.

In analoger Weise können auch die Ausdrücke für die Quervergrößerung, die Dispersion und den Öffnungsfehler eines Magnetfeldes mit Streufeld auf die entsprechenden Ausdrücke des homogenen Feldteiles zurückgeführt werden.

Das Kernstück der Ableitung bildet die Berechnung der Bahnen im homogenen Feldteil (vgl. Abb. 10 u. 11). Es wird ein Hauptstrahl und ein demselben benachbarter Nebenstrahl betrachtet. Der Hauptstrahl trete unter dem Winkel $\varepsilon_{10}$ gegen die Normale zur Feldgrenze in den homogenen Teil des Sektorfeldes ein und unter dem Winkel $\varepsilon_{20}$ gegen die Normale zur Feldgrenze aus diesem wieder aus. $\varepsilon_{10}$ und $\varepsilon_{20}$ sollen positiv gezählt werden, wenn die Normale auf der dem Ablenkungszentrum abgewandten Seite des Hauptstrahles liegt. Im homogenen Teil des Sektorfeldes erfolge eine Ablenkung um $\Phi_{0}$. Der Bahnradius sei dort $r_{\mathrm{m}}$. Der Nebenstrahl besitze den Bahnradius $r_{\mathrm{m}}(1+\varrho)(\varrho \ll 1)$. Er trete mit einer Neigung um $\alpha_{10}$ gegen den Hauptstrahl in das homogene Sektorfeld ein und mit einer Neigung $\alpha_{20}$ gegen den Hauptstrahl aus dem homogenen Teil des Sektorfeldes aus. Die Abstände auf den Tangenten an die Grenze des homogenen Feldes in Ein- bzw. Austrittspunkt des Hauptstrahles zwischen Haupt- und Nebenstrahl seien $d_{1}$ bzw. $d_{2} . d_{i}$ soll positiv gezählt werden, wenn der Nebenstrahl auf der dem Ablenkungszentrum abgewandten Seite des Hauptstrahles liegt.

$d_{2}$ und $\alpha_{20}$ sind von $\alpha_{10}, \varrho$ und $d_{1}$ abhängig. Man findet in zweiter Näherung

$$
\begin{aligned}
d_{2}=P_{1} \alpha_{10}+ & P_{2} \varrho+P_{3} d_{1}+P_{11} \alpha_{10}{ }^{2}+P_{12} \alpha_{10} \varrho \\
& +P_{13} \alpha_{10} d_{1}+P_{22} \varrho^{2}+P_{23} \varrho d_{1}+P_{33} d_{1}{ }^{2}
\end{aligned}
$$




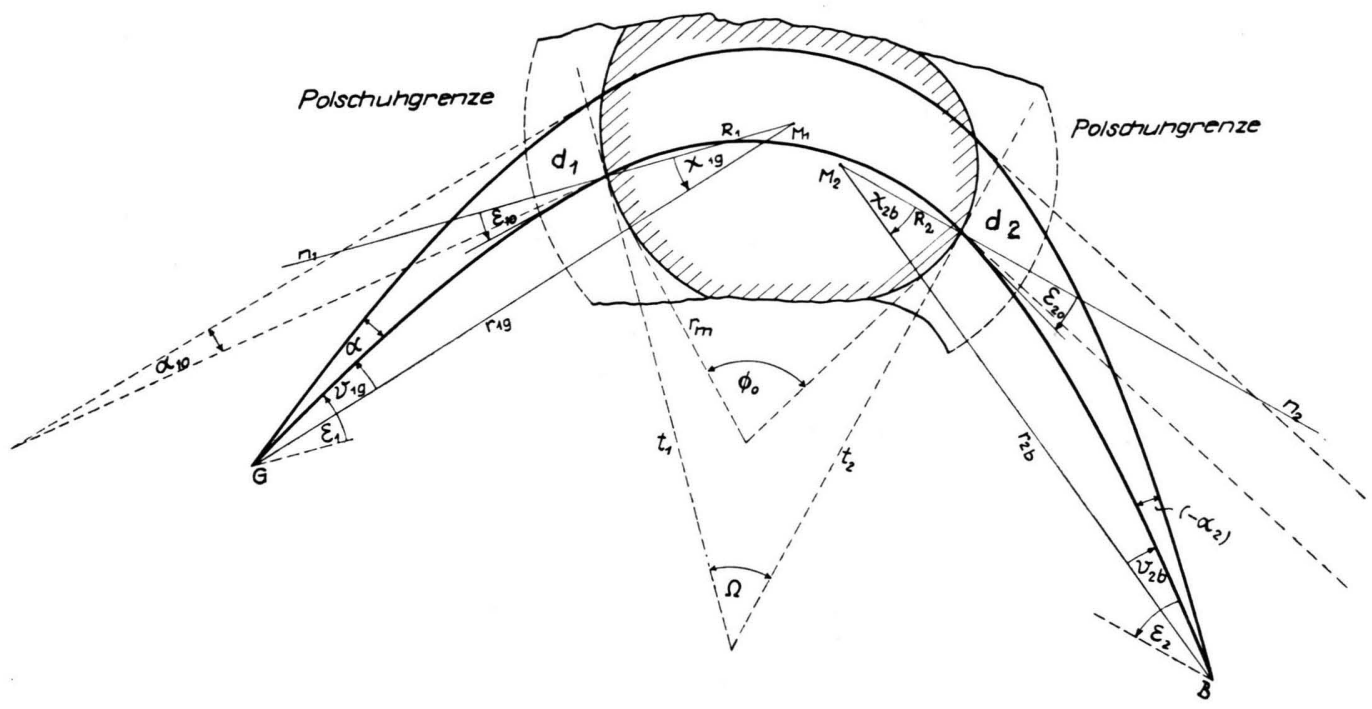

Abb. 11. Strahlengang bei gekrümmter Polschuhbegrenzung.

mit

$$
\begin{aligned}
& P_{1}=r_{\mathrm{m}} \frac{\sin \Phi_{0}}{\cos \varepsilon_{20}}, \\
& P_{2}=r_{\mathrm{m}} \frac{1-\cos \Phi_{0}}{\cos \varepsilon_{20}}, \\
& P_{3}=\frac{\cos \left(\Phi_{0}-\varepsilon_{10}\right)}{\cos \varepsilon_{20}}, \\
& P_{11}=\frac{r_{\mathrm{m}}}{2 \cos \varepsilon_{20}}\left[\cos \Phi_{0}-\frac{\cos ^{2}\left(\Phi_{0}-\varepsilon_{20}\right)}{\cos ^{2} \varepsilon_{20}}\right], \\
& P_{13}=\frac{\cos \left(\Phi_{0}-\varepsilon_{20}\right) \sin \Omega}{\cos ^{3} \varepsilon_{20}}, \\
& P_{33}=-\frac{\sin ^{2} \Omega}{2 r_{\mathrm{m}} \cos ^{3} \varepsilon_{20}}+\frac{P_{1}}{2 r_{\mathrm{m}} R_{1} \cos \varepsilon_{10}}
\end{aligned}
$$

und

$$
\begin{aligned}
& \alpha_{20}=Q_{1} \alpha_{10}+Q_{2} \varrho+Q_{3} d_{1}+Q_{11} \alpha_{10}{ }^{2}+Q_{12} \alpha_{10} \varrho \\
& \quad+Q_{13} \alpha_{10} d_{1}+Q_{22} \varrho^{2}+Q_{23} \varrho d_{1}+Q_{33} d_{1}{ }^{2}+Q_{44} d_{2}{ }^{2}
\end{aligned}
$$

mit

$$
\begin{aligned}
& Q_{1}=\frac{\cos \left(\Phi_{0}-\varepsilon_{20}\right)}{\cos \varepsilon_{20}}, \\
& Q_{2}=\sin \Phi_{0}+\operatorname{tg} \varepsilon_{20}\left(1-\cos \Phi_{0}\right), \\
& Q_{3}=-\frac{\sin \Omega}{r_{\mathrm{m}} \cos \varepsilon_{20}}, \\
& Q_{11}=-\frac{1}{2}\left[\frac{\sin \left(\Phi_{0}-\varepsilon_{20}\right)}{\cos \varepsilon_{20}}+\frac{\cos ^{2}\left(\Phi_{0}-\varepsilon_{20}\right) \sin \varepsilon_{20}}{\cos ^{3} \varepsilon_{20}}\right], \\
& Q_{13}=\frac{1}{r_{\mathrm{m}}} \frac{\cos \left(\Phi_{0}-\varepsilon_{20}\right) \sin \varepsilon_{20} \sin \Omega}{\cos ^{3} \varepsilon_{20}}, \\
& Q_{33}=-\frac{1}{2 r_{\mathrm{m}}^{2}} \frac{\sin \varepsilon_{20} \sin ^{2} \Omega}{\cos ^{3} \varepsilon_{20}}+\frac{Q_{1}}{2 r_{\mathrm{m}} R_{1} \cos \varepsilon_{10}}, \\
& Q_{44}=\frac{1}{2 r_{\mathrm{m}} R_{2} \cos \varepsilon_{20}} .
\end{aligned}
$$

Die nicht angegebenen Koeffizienten $P, Q$ werden im folgenden nicht benötigt.

Zur Berechnung der Gesamtablenkung hat man auch die Bahngleichungen im Streufeld zu beachten. Diese lauten ${ }^{14}$ im Spezialfall 1

$$
y_{\mathrm{B}}(z)=y_{\mathrm{B} 0}+\int_{z_{0}}^{z} \frac{f(\xi) \mathrm{d} \xi}{\left(1-f^{2}(\xi)\right)^{1 / 2}}
$$

mit $f(z)=\sin \varepsilon(z)=-\frac{1}{r_{\mathrm{m}}} \int_{z_{0}}^{z} h(\xi) \mathrm{d} \xi+\sin \varepsilon_{0}$

und im Spezialfall 2

$$
\chi_{\mathrm{B}}(r)=\chi_{\mathrm{B} 0}+\int_{r_{0}}^{r} \frac{1}{\varrho} \frac{f(\varrho) \mathrm{d} \varrho}{\left(\varrho^{2}-f^{2}(\varrho)\right)^{1 / 2}}
$$

mit $f(r)=r \sin v(r)=\frac{1}{r_{\mathrm{m}}} \int_{r_{0}}^{r} h(\varrho) \varrho \mathrm{d} \varrho+r_{0} \sin \nu_{0}$.

Zur Berechnung des Öffnungsfehlers sind auf der Gegenstandsseite $d_{1}$ und $\alpha_{10}$ durch den halben Öffnungswinkel $\alpha$ der abbildenden Strahlenbündel auszudrücken. Hierbei ist $\varrho=0$ vorauszusetzen. Zur Berechnung der Lateralvergrößerung $V$ sind $d_{1}$ und $\alpha_{10}$ durch die Gegenstandsgröße $b_{1}$ auszudrücken, wobei $\alpha=0$ und $\varrho=0$ zu setzen ist. Zur Berechnung der Dispersion ist $\alpha=0$ zu setzen sowie $\alpha_{10}$ und $d_{1}$ durch $\varrho$ auszudrücken.

${ }_{14}$ N. D. C o g g e s h a 11 u. M. M u s k a t, Phys. Rev. 66, 187 [1944]. 
Der Ausdruck für die Lateralvergrößerung lautet ${ }^{1}$

$$
V=-\frac{A_{2}}{A_{1}} \frac{l_{2}{ }^{*}-g_{20}}{f_{0}}
$$

wobei die Größen $A_{i}$ durch die Gln. (18) und (20) gegeben sind. - Für die Dispersion erhält man bei geraden Feldgrenzen

$$
\begin{aligned}
& K_{\mathrm{m}}=\frac{\cos \varepsilon_{2}}{\cos \varepsilon_{20}}\left\{r_{\mathrm{m}}\left(1-\cos \Phi_{0}\right)+l_{2}{ }^{*}\left[\sin \Phi_{0}+\operatorname{tg} \varepsilon_{20}\left(1-\cos \Phi_{0}\right)\right]\right. \\
& \left.+\frac{l_{2}^{*}-g_{20}}{f_{0}}\left[\frac{l_{1}^{*}}{r_{\mathrm{m}} \cos \varepsilon_{10}} \int_{-z_{1 \mathrm{~g}}}^{0} h_{1}(\xi) \mathrm{d} \xi-\frac{\cos \varepsilon_{10}}{r_{\mathrm{m}}} \int_{-z_{1 \mathrm{~g}}}^{0} \frac{\int_{-z_{1 \mathrm{~g}}}^{\xi} h_{1}\left(\xi^{\prime}\right) \mathrm{d} \xi^{\prime}}{\left(1-f_{1}{ }^{2}(\xi)\right)^{3 / 2}} \mathrm{~d} \xi\right]+\frac{\cos \varepsilon_{20}}{r_{\mathrm{m}}} \int_{0}^{z_{2 \mathrm{~b}}} \frac{\int_{0}^{\xi} h_{2}\left(\xi^{\prime}\right) \mathrm{d} \xi^{\prime}}{\left(1-f_{2}{ }^{2}(\xi)\right)^{3 / 2}} \mathrm{~d} \xi\right\} .
\end{aligned}
$$

Für gekrümmte Feldgrenzen erhält man

$K_{\mathrm{m}}=\frac{r_{2 \mathrm{~b}} \cos \nu_{2 \mathrm{~b}}}{l_{2}{ }^{*}+R_{2} \cos \varepsilon_{20}}\left\{r_{\mathrm{m}}\left(1-\cos \Phi_{0}\right)+l_{2}{ }^{*}\left[\sin \Phi_{0}+\operatorname{tg} \varepsilon_{20}\left(1-\cos \Phi_{0}\right)\right]+\frac{l_{2}^{*}-g_{20}}{f_{0}}\right.$

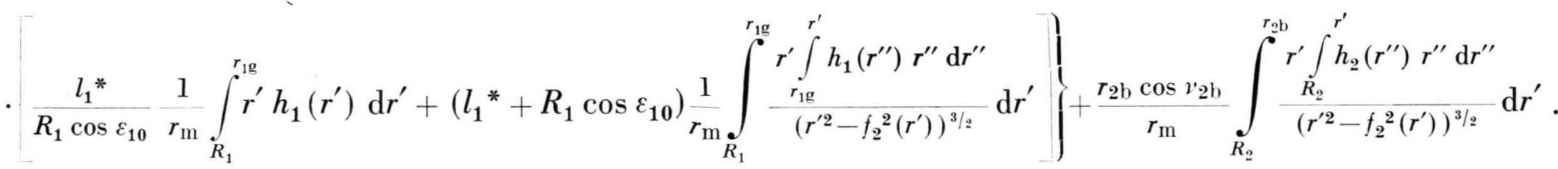

Wird das Streufeld vernachlässigt, so bleibt in diesen Gleichungen für $K_{\mathrm{m}}$ jeweils nur der bekannte, in der ersten Zeile stehende Ausdruck für die Dispersion des homogenen Magnetfeldes übrig.

Für den Öffnungsfehler erhält man

$$
\Delta B=-{ }^{1 / 2} A_{1}{ }^{2} A_{2}\left\{B_{1} \sqrt{K}+B_{2} / K\right\} \alpha^{2} .
$$

$K$ ist eine Abkürzung für den Ausdruck:

$K=\left(\frac{l_{2}{ }^{*}-g_{20}}{f_{0}}\right)^{2}=\frac{l_{2} *-g_{20}}{l_{1}^{*}-g_{10}}=\frac{l_{2}{ }^{* 2}+\left[r_{\mathrm{m}}+l_{2}{ }^{*} \operatorname{tg} \varepsilon_{20}\right]^{2}}{l_{1}^{* 2}+\left[r_{\mathrm{m}}+l_{1}{ }^{*} \operatorname{tg} \varepsilon_{10}\right]^{2}} ;$

die Größen $g_{i 0}$ und $f_{0}$ wurden in Gl. $(2 \mathrm{a}, \mathrm{b})$ und Gl. (2c) angegeben.

Für gerade Feldgrenzen kann $l_{i}^{*}$ aus Gl. (4) entnommen werden, die Größen $A_{i}$ und $B_{i}$ sind gegeben durch

$$
\begin{aligned}
& A_{i}=\frac{\cos \varepsilon_{i}}{\cos \varepsilon_{i 0}}, \\
& B_{i}=r_{\mathrm{m}}+3(-1)^{i+1} \cos ^{3} \varepsilon_{i 0} \int_{a_{i}}^{b_{i}} \frac{f_{i}(\xi) \mathrm{d} \xi}{\left(1-f_{i}{ }^{2}(\xi)\right)^{5 / 2}}
\end{aligned}
$$

mit $\quad a_{1}=-z_{1 \mathrm{~g}}, b_{1}=0 ; a_{2}=0, b_{2}=z_{2 \mathrm{~b}}$,

$$
f_{i}(\zeta)=-\frac{1}{r_{\mathrm{m}}} \int_{0}^{\zeta} h_{i}\left(\zeta^{\prime}\right) \mathrm{d} \zeta^{\prime}+(-1)^{i+1} \sin \varepsilon_{i 0} .
$$

Für gekrümmte Feldgrenzen folgt $l_{i}^{*}$ aus Gl. (6) und die Größen $A_{i}$ und $B_{i}$ sind

$$
\begin{gathered}
A_{i}=\frac{r_{i 0} \cos \gamma_{i}}{l_{i}^{*}+R_{i} \cos \varepsilon_{i 0}} \\
\left(r_{10}=r_{1 \mathrm{~g}}, v_{1}=v_{1 \mathrm{~g}} ; r_{20}=r_{2 \mathrm{~b}}, v_{2}=v_{2 \mathrm{~b}}\right), \\
B_{i}=r_{\mathrm{m}}+l_{i}^{*} \operatorname{tg} \varepsilon_{i 0}\left[1-\left(1+\frac{l_{i}^{*}}{R_{i} \cos \varepsilon_{i 0}}\right)^{2}\right]-\frac{\sin \varepsilon_{i 0}}{\cos ^{2} \varepsilon_{i 0}} \frac{l_{i}^{* 2}}{R_{i}} \\
-\frac{l_{i}^{* 3}}{r_{\mathrm{m}} R_{i} \cos ^{3} \varepsilon_{i 0}}+3\left(l_{i}^{*}+R_{i} \cos \varepsilon_{i 0}\right)^{3} \int_{R_{i}}^{r_{i 0}} \frac{\varrho f_{i}(\varrho) \mathrm{d} \varrho}{\left(\varrho^{2}-f_{i}{ }^{2}(\varrho)\right)^{5 / e}}
\end{gathered}
$$

mit $\quad f_{i}(\varrho)=\frac{1}{r_{\mathrm{m}}} \int_{R_{i}}^{\varrho} h_{i}\left(\varrho^{\prime}\right) \varrho^{\prime} \mathrm{d} \varrho^{\prime}+R_{i} \sin \varepsilon_{i 0}$.

Wird das Streufeld vernachlässigt, so wird $\varepsilon_{i 0}=\varepsilon_{i}$, $l_{i}^{*}=l_{i}, A_{i}=1$ und

$$
\text { - } B_{i}=r_{\mathrm{m}}+3 l_{i} \operatorname{tg} \varepsilon_{i}-\frac{l_{i}{ }^{3}}{r_{\mathrm{m}} R_{i} \cos ^{3} \varepsilon_{i}} .
$$

Der Öffnungsfehler wird dann

$\triangle B=$

$-\frac{1}{2}\left\{\left(r_{\mathrm{m}}+3 l_{1} \operatorname{tg} \varepsilon_{1}-\frac{l_{1}{ }^{3}}{r_{\mathrm{m}} R_{1} \cos ^{3} \varepsilon_{1}}\right)\left(\frac{l_{2}{ }^{2}+\left[r_{\mathrm{m}}+l_{2} \operatorname{tg} \varepsilon_{2}\right]^{2}}{l_{1}{ }^{2}+\left[r_{\mathrm{m}}+l_{1} \operatorname{tg} \varepsilon_{1}\right]^{2}}\right)^{\frac{1}{2}}\right.$

$\left.+\left(r_{\mathrm{m}}+3 l_{2} \operatorname{tg} \varepsilon_{2}-\frac{l_{2}{ }^{3}}{r_{\mathrm{m}} R_{2} \cos ^{3} \varepsilon_{2}}\right) \frac{l_{1}^{2}+\left[r_{\mathrm{m}}+l_{1} \operatorname{tg} \varepsilon_{1}\right]^{2}}{l_{2}^{2}+\left[r_{\mathrm{m}}+l_{2} \operatorname{tg} \varepsilon_{2}\right]^{2}}\right\} \alpha^{2}$.

Dieser Ausdruck ist mit dem von dem einen von uns (H. H.) bereits früher ${ }^{15}$ angegebenen Ausdruck für den Öffnungsfehler des homogenen Sektorfeldes 
identisch. Gl. (21) gilt für gekrümmte Feldgrenzen. Man erhält daraus für $R_{i}=\infty$ den Öffnungsfehler für gerade Feldgrenzen.

Bei der Durchführung der Rechnungen wurden die trigonometrischen Identitäten

$\sin \left(\Phi_{0}-\varepsilon_{10}\right) \cos \varepsilon_{20}-\cos \left(\Phi_{0}-\varepsilon_{10}\right) \sin \varepsilon_{20}=\sin \Omega$,

$\sin \left(\Phi_{0}-\varepsilon_{20}\right) \cos \left(\Phi_{0}-\varepsilon_{10}\right)$

$$
-\sin \varepsilon_{10} \cos \varepsilon_{20}=\cos \Phi_{0} \sin \Omega \text {, }
$$

$\cos \varepsilon_{10} \cos \varepsilon_{20}$

$$
-\cos \left(\Phi_{0}-\varepsilon_{10}\right) \cos \left(\Phi_{0}-\varepsilon_{20}\right)=\sin \Phi_{0} \sin \Omega
$$

benutzt. Ferner wurde mehrfach die Linsengl. (1) angewandt, wodurch erst eine übersichtlichere Darstellung der Ergebnisse möglich wurde.

\section{Anwendung}

Die Nützlichkeit der angegebenen Linsengleichung sowie der für Lateralvergrößerung, Dispersion und Öffnungsfehler abgeleiteten Formeln soll durch ihre Anwendung auf Apparate mit symmetrischem Strahlengang im Magnetfeld, bei denen die Bahntangenten im Gegenstands- und Bildort auf den ebenen Polschuhstirnflächen senkrecht stehen $\left(\varepsilon_{1}=\varepsilon_{2}=0\right)$, demonstriert werden. Man erhält bei der Spezialisierung auf symmetrischen Strahlengang, wenn man gleichen Feldabfall auf Gegenstands- und Bildseite voraussetzt: $l^{*}=f_{0}+g_{0}, V=-1$,

$$
\begin{aligned}
& K_{\mathrm{m}}=2 r_{\mathrm{m}}\left\{\frac{\sin \left(\Phi_{0}-\varepsilon_{0}\right)+\sin \varepsilon_{0}}{\sin \left(\Phi_{0}-2 \varepsilon_{0}\right)}\right.
\end{aligned}
$$

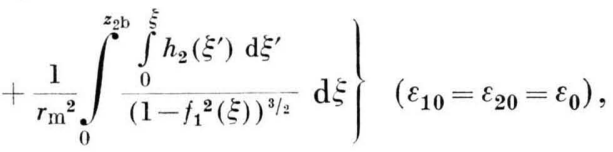

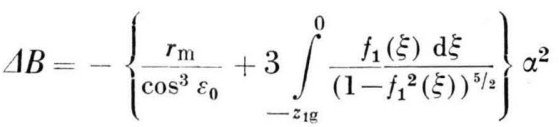

mit $f_{1}(\xi)=-\frac{1}{r_{\mathrm{m}}} \int_{z_{1 \mathrm{~g}}}^{\xi} h_{1}(\zeta) \mathrm{d} \zeta$. Da nun $f_{1}$ eine kleine

Größe darstellt, erhält man durch Entwickeln nach Potenzen von $f_{1}$, wenn man als Integrationsgrenze

15 H. Hint enberger, Z. Naturforschg. 3 a, 125 [1948]. 3 a, 669 [1948]; 6a, 275 [1951]; Rev. Sci. Instrum. 20, 748 [1949]. $z_{1 \mathrm{~g}}$ den Wert $l$ bei fehlendem Streufeld und gleicher Gesamtablenkung einsetzt:

$$
\begin{gathered}
z_{1 \mathrm{~g}}-l=k \int_{-\frac{l}{k}}^{0} h_{1}\left(\frac{\xi}{k}\right) \mathrm{d}\left(\frac{\xi}{k}\right)+0\left(\frac{k}{\varkappa^{2}}\right), \\
K_{\mathrm{m}}=2 r_{\mathrm{m}}\left[1-\frac{1}{\varkappa} \cot \frac{\Omega}{2} \int_{-i / k}^{0} h_{1}\left(\frac{\xi}{k}\right) \mathrm{d}\left(\frac{\xi}{k}\right)\right], \\
\Delta B=-r_{\mathrm{m}}\left\{1+\frac{3}{2 \varkappa^{2}}\left[\left(\int_{-i / k}^{0} h_{1}\left(\frac{\xi}{k}\right) \mathrm{d}\left(\frac{\xi}{k}\right)\right)^{2}\right.\right. \\
\left.\left.-2 \int_{-i / k}^{0} \int_{-i / k}^{\xi / k} h_{1}\left(\frac{\xi^{\prime}}{k}\right) \mathrm{d}\left(\frac{\xi^{\prime}}{k}\right) \mathrm{d}\left(\frac{\xi}{k}\right)\right]\right\},
\end{gathered}
$$

worin $\varkappa=r_{\mathrm{m}} / k$ bedeutet. Gl. (25) stellt die mathematische Formulierung der altbekannten Aussage dar, daß das Streufeld in erster Näherung durch ein homogenes Feld ersetzt werden darf, in dem eine Richtungsänderung um denselben Betrag erfolgt. Nach Gl. (12) gilt nämlich

$$
\Delta \sin \varepsilon=-\frac{1}{r_{\mathrm{m}}} \int_{a}^{b} h(z) \mathrm{d} z=-\frac{1}{r_{\mathrm{m}}} \int_{a}^{b^{*}} 1 \mathrm{~d} z,
$$

worin $b^{*}-a \equiv \xi$ die Ausdehnung des homogenen Ersatzfeldes darstellt, das die gleiche Ablenkung wie das von $a$ bis $b$ reichende Streufeld bewirkt. Diese Beziehung läßt sich nach $\xi$ auflösen, wobei sich das bekannte Ergebnis

$$
\xi=\int_{a}^{b} h(z) \mathrm{d} z
$$

ergibt. (Gleichung (29) gilt in erster Näherung auch für schrägen Eintritt.) Wendet man dieses Resultat auf das ohne Blende gemessene Streufeld an (s. Abb. 5), so findet man $\xi=3,6 k$. Da zur Berechnung die Grenze zwischen Streufeld und homogenem Feld bei $Z_{\mathrm{s}}=-1,5 k$ angenommen worden war, so liegt also die Ersatzfeldgrenze bei $Z_{\mathrm{E}}=+2,1 k$ vor dem Polschuhrand. Aus einer nicht publizierten Messung der Lage von Gegenstands- und Bildort an einem Massenspektrometer mit einem Magneten vom Typ A (Abb. 3) von $\mathrm{H}$ in te $\mathrm{nberger}$ und $\mathrm{L}$ ang hatte sich $Z_{\mathrm{E}}=2,2 k$ ergeben. Nach einer von $\mathrm{N}$ i e r ${ }^{16}$ angegebenen Regel wäre $Z_{\mathrm{E}}=2,0 k$. Die Übereinstimung dieser drei Werte ist also recht gut. Von

${ }^{16}$ A. O. N i e r, Rev. Sci. Instrum. 11, 212 [1940]. 
anderen Autoren $\left(\mathrm{Ploch}\right.$ und $\mathrm{Walcher}{ }^{7}$, Bainbridge ${ }^{17}$ ) wurden jedoch Messungen durchgeführt, die zu hiervon abweichenden Ergebnissen führten. Dies ist aber keineswegs überraschend, da der Feldabfall und damit $\xi$ von der Gestalt der Polschuhstirnfläche abhängt.

Tab. 1 gibt die durch das Streufeld verursachten Änderungen der Dispersion und des Öffnungsfehlers für einige Magnetfelder in gebräuchlichen Apparaten wieder.

\begin{tabular}{|c|c|c|c|c|}
\hline & $\Omega=\Phi$ & $\varkappa$ & $\frac{K_{\mathrm{m}}-\left(K_{\mathrm{m}}\right)_{0}}{\left(K_{\mathrm{m}}\right)_{0}}$ & $\frac{\Delta B-(\Delta B)_{0}}{(\Delta B)_{0}}$ \\
\hline 1 & $60^{\circ}$ & 159 & $-4^{\%} \%$ & $-10 \%$ \\
2 & $60^{\circ}$ & 20 & $-31^{\%} \%$ & $-6,5^{\%}$ \\
3 & $90^{\circ}$ & 20 & $-18^{\circ} \%$ & $-6,5 \%$ \\
\hline
\end{tabular}

Tab. 1. 1. Massenspektrograph von $\mathrm{B}$ a in brid g e ${ }^{18}$; 2. und 3. gebräuchliche Massenspektrometer. Die mit dem Index 0 versehenen Größen beziehen sich auf das ideale homogene Sektorfeld mit gleichem Bahnradius $r_{\mathrm{m}}$, gleicher Gesamtablenkung $\Phi$ und gleichem Ein- und Austrittswinkel $\varepsilon_{i}$.

Bei der Berechnung von Tab. 1 waren die Zahlenwerte

$$
\begin{gathered}
\int_{-\frac{l}{k}}^{0} h_{1}\left(\frac{\xi}{k}\right) \mathrm{d}\left(\frac{\xi}{k}\right)=3,6, \\
\frac{3}{2}\left[\left(\int_{-\frac{l}{k}}^{0} h_{1}\left(\frac{\xi}{k}\right) \mathrm{d}\left(\frac{\xi}{k}\right)\right)^{2}-2 \int_{-\frac{l}{k}}^{0} \int_{-\frac{l}{k}}^{\xi^{k}} h_{1}\left(\frac{\xi}{k}\right) \mathrm{d}\left(\frac{\xi}{k}\right) \mathrm{d}\left(\frac{\xi}{k}\right)\right] \\
=-26,1
\end{gathered}
$$

berücksichtigt worden.

In der Praxis interessiert weniger $z_{1 \mathrm{~g}}$ als vielmehr der Abstand $L$ von Gegenstand und Bild vom Pol-

17 K. T. B a in bridg e, Phys. Rev. 75, 216 [1949]. schuhrand. Man erhält $L$, indem man den Abstand $\left|Z_{\mathrm{s}}\right|=1,5 k$ der Grenze zwischen Streufeld und homogenem Feld vom Polschuhrand von $z_{1 \mathrm{~g}}$ subtrahiert. Der Berechnung der obenerwähnten Integrale war die Grenze zwischen Streufeld und homogenem Feld bei $Z=-1,5 k$ zugrunde gelegt worden, wodurch $L=l+2,1 k$ wird.

Wie man sieht, sind die Korrekturen sehr stark von der Dimensionierung des Magneten abhängig. Sie betragen jedoch für die in doppelfokussierenden Massenspektrographen üblichen Magneten nur wenige Prozent, während sie für andere Magnete sehr beträchtlich werden können.

Es muß darauf hingewiesen werden, daß die nicht in der Symmetrieebene verlaufenden Bahnen die Linienbreite vergrößern, so daß diese nicht nur von $V$ und $\Delta B$ abhängt. Durch Anwendung von Blenden, die den Abstand der zur Abbildung beitragenden Strahlen von der Mittelebene begrenzen, läßt sich dieser Effekt jedoch beliebig verringern. Eine solche Beschränkung auf Strahlen in der unmittelbaren Umgebung der Mittelebene ist z. B. bei massenspektrographischen Dublettmessungen auch aus anderen Gründen notwendig.

Die in dieser Arbeit angegebenen Ergebnisse lassen sich nicht immer unmittelbar mit denen der anderen Autoren vergleichen. Es ist vielmehr bei einer Gegenüberstellung des idealen Falles (kein Streufeld) und des allgemeinen Falles (Streufeld) darauf zu achten, welcher von den Parametern $\Phi, \Phi_{0}, r_{\mathrm{m}}, \varepsilon_{i}$ hierbei konstant gehalten wird. Von der Wahl dieser Parameter hängt nämlich die Größe der Korrekturen ab. (In der vorliegenden Arbeit wurden $\Phi$, $r_{\mathrm{m}}$ und $\varepsilon_{i}$ konstant gehalten. Die Größen $\Phi_{0}, \varepsilon_{10}$ und $\varepsilon_{20}$ dagegen (s. Abb. 10 u. 11) sind im Ersatzfeld und im wirklichen Feld voneinander verschieden.)

${ }^{18}$ K. T. B a in bri d g e u. E. B. J or d a n, Phys. Rev. 50, 282 [1936]. 